\title{
An Antibody exo Diels-Alderase Inhibitor Complex at 1.95 Angstrom Resolution
}

\author{
Andreas Heine, Enrico A. Stura, Jari T. Yli-Kauhaluoma, \\ Changshou Gao, Qiaolin Deng, Brett R. Beno, Kendall N. Houk, \\ Kim D. Janda, Ian A. Wilson
}

A highly specific Diels-Alder protein catalyst was made by manipulating the antibody repertoire of the immune system. The catalytic antibody $13 \mathrm{G} 5$ catalyzes a disfavored exo Diels-Alder transformation in a reaction for which there is no natural enzyme counterpart and that yields a single regioisomer in high enantiomeric excess. The crystal structure of the antibody Fab in complex with a ferrocenyl inhibitor containing the essential haptenic core that elicited 13G5 was determined at 1.95 angstrom resolution. Three key antibody residues appear to be responsible for the observed catalysis and product control. Tyrosine-L36 acts as a Lewis acid activating the dienophile for nucleophilic attack, and asparagine-L91 and aspartic acid-H50 form hydrogen bonds to the carboxylate side chain that substitutes for the carbamate diene substrate. This hydrogenbonding scheme leads to rate acceleration and also pronounced stereoselectivity. Docking experiments with the four possible ortho transition states of the reaction explain the specific exo effect and suggest that the $(3 R, 4 R)$-exo stereoisomer is the preferred product.

The Diels-Alder reaction is the quintessential pericyclic transformation in organic chemistry (1). The reaction of a diene with a dienophile provides enormous opportunities to generate cyclic structures. This reaction is facilitated by the presence of electron-donating groups on the diene and electron-withdrawing functionalities on the dienophile (2). Detailed studies of Diels-Alder reaction rates have shown some dependency on the solvent (3); for example, in aqueous solution, the Diels-Alder reaction is often accelerated as a consequence of hydrogen bonding of water to the dienophile in the transition state. Because of its synthetic importance, substantial interest has arisen in the theoretical aspects of this reaction. Various reaction schemes have been proposed that range from biradical to synchronous bond formation (2).

The immune system has been shown to generate various de novo antibody catalysts (4). The application of transition state theory in hapten design (5), based on the reaction mechanism, led to the initial production of esterolytic antibodies (6). Further advances in transition state analog design, combined with "bait and switch" (4) and the strategy of reactive immunization (7), have resulted in an impressive array of catalytic antibodies that can effect a wide range of chemical transformations (4).

A. Heine, E. A Stura, J. T. Yli-Kauhaluoma, C. Gao, K. D. Janda, I. A. Wilson, The Skaggs Institute of Chemical Biology and the Departments of Molecular Biology and Chemistry. The Scripps Research Institute, 10550 North Torrey Pines Road, La Jolla, CA 92037, USA.

Q. Deng, B. R. Beno, K. N. Houk, Department of Chemistry and Biochemistry, University of California, Los Angeles, CA 90095, USA.
A desirable goal has been the generation of antibody catalysts for which enzymatic counterparts are not available (8). The existence of a natural Diels-Alderase enzyme has been proposed (9), but its function and catalytic mechanism have not yet been characterized. Structural studies of antibody-hapten complexes can supply information on how such reactions are catalyzed and allow comparison with natural enzymes, when they exist, as well as suggest how mutations might improve their catalytic rates. These principles were aptly demonstrated with the catalytic antibody structure of 1F7 (10), which converts chorismate to prephenate in a pericyclic process related to the Diels-Alder reaction.

The exo Diels-Alder reaction forms a trans cyclohexene adduct 3 from 4-carboxybenzyl trans-1,3-butadiene-1-carbamate (1) with $N, N$-dimethylacrylamide (2) (Fig. 1). This Diels-Alder reaction is disfavored relative to the formation of the endo-cis adduct 4. Two approaches have been used to generate antibodies capable of catalyzing the Diels-Alder reaction. The first used conventional, rigid, bicyclic transition state analogs to elicit antibody catalysts (11), in which the distribution of products could be controlled for the reaction between the diene and dienophile (Fig. 1). With this strategy, specific endo and exo Diels-Alder antibodies were selected with bicyclic haptens 5 and 6 (12). A more radical approach in hapten design

Fig. 1. A disfavored exo Diels-Alder reaction catalyzed by antibody 13G5. The 4-carboxybenzyl trans-1,3-butadiene-1-carbamate $\mathbf{1}$ reacts with $\mathrm{N}, \mathrm{N}$-dimethylacrylamide 2 to form the cyclohexene product 3 (Me, methyl). The less energetically favorable exo product 3 is formed rather than the endo product 4 . The inhibitor 1-carboxy-1'[(dimethylamino)carbonyl] ferrocene $\mathbf{9}$ is the core structure of the original hapten and was used for both antibody-hapten affinity measurements (13) and in our crystallographic studies because this compound is more chemically stable. The 1-[(4carboxy-1-oxobutyl)amino]-1'-[(dimethylamino)carbonyl]ferrocene complex 7 was linked to keyhole limpet hemocyanin
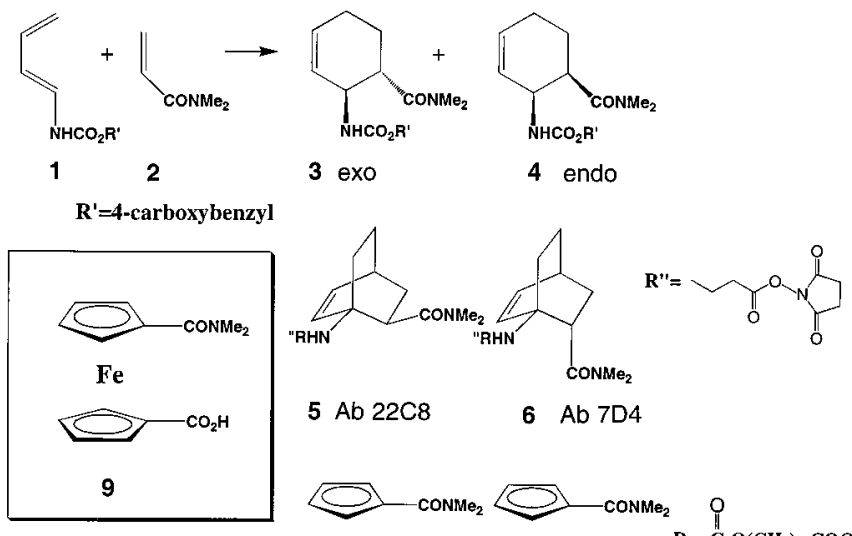

6 Ab 7D4

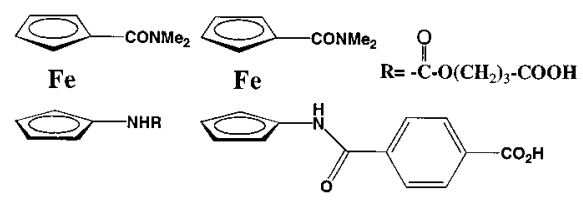

7 Ab $13 G 5$

8 Ab 4D5

\begin{tabular}{|c|c|l|l|l|l|c|}
\hline Hapten & Antibody & endo:exo, (ee) $\{\%\}$ & $\begin{array}{l}K_{\mathrm{M}} \\
(\mathrm{diene}) \\
(\mathrm{mM})\end{array}$ & $\begin{array}{l}K_{\mathrm{M}} \\
(\mathrm{dienophile}) \\
(\mathrm{mM})\end{array}$ & $K_{\text {cat }}\left(\mathrm{min}^{-1}\right)$ & $\begin{array}{l}\text { E.M. } \\
(\mathrm{M})\end{array}$ \\
\hline Uncat. & & $85: 15,(50)$ & & & & \\
\hline 5 & $22 \mathrm{C} 8$ & complete exo, $(>98)$ & 0.70 & 7.5 & $3.17 \times 10^{-3}$ & 18 \\
\hline 6 & $7 \mathrm{D} 4$ & complete endo, $(>98)$ & 0.96 & 1.7 & $3.44 \times 10^{-3}$ & 4.8 \\
\hline 7 & $13 \mathrm{G} 5$ & $>98 \%$ exo, $(95)$ & 2.7 & 10 & $1.20 \times 10^{-3}$ & 6.9 \\
\hline 8 & $4 \mathrm{D} 5$ & $>98 \%$ endo, $(95)$ & 1.6 & 5.9 & $3.48 \times 10^{-3}$ & 4.9 \\
\hline
\end{tabular}
and used as a hapten to

elicit 13G5. The constrained bicyclo[2.2.2]octene hapten $\mathbf{5}$ was originally chosen because it is a close mimic of the transition state in the Diels-Alder reaction (11). The product distribution, Michaelis constant $\left(K_{\mathrm{m}}\right)$, catalytic rate constant $\left(k_{\mathrm{cat}}\right)$, and rate enhancement [expressed as effective molarity (E.M.)], for the catalytic antibodies generated with 5 and $\mathbf{6}$ (12) and with ferrocenyl compounds $\mathbf{7}$ and $\mathbf{8}$ (13) are compared in the table. Uncat., uncatalyzed. 
resulted from the use of a ferrocene derivative 7 as a transition state analog for antibody generation. This hapten is highly flexible, with the cyclopentadienyl rings able to rotate freely in solution. We chose this hapten to test whether the immune system can select a conformer that mimics one of the Diels-Alder transition states and, hence, produce a specific Diels-Alderase (13), and to challenge the concept that the best haptens for reactions that proceed through highly ordered transition states must be conformationally restricted. Antibody 13G5 was raised to 7, which had been linked through its carboxylic acid group as an amide to keyhole limpet hemocyanin. The antibody catalyzes the disfavored exo Diels-Alder reaction of substrates 1 and 2 (Fig. 1) (13) and catalyzes the formation of the ortho product with high regio-, diastereo-, and enantioselectivity, forming only one out of eight possible products. In comparison, the uncatalyzed reaction of these substrates is only regioselective, with a ratio of ortho-endo to ortho-exo products of $85: 15$. The ortho transition state is predicted to be 2 to 4 $\mathrm{kcal} / \mathrm{mol}$ lower in energy than the meta transition state, with the endo transition states being about $1.9 \mathrm{kcal} / \mathrm{mol}$ lower in energy than the exo transition states (12). In the uncatalyzed reaction, no enantiomeric preference was observed (Fig. 1).

We report here crystallographic studies on a complex of this catalytic antibody with the more stable inhibitor 9, which was also used for antibody-hapten affinity measurements (13). We also determined the crystal structure of the unbound inhibitor and performed quantum mechanical calculations to probe the catalytic mechanism of this DielsAlder reaction. Docking of the four possible ortho transition states revealed the basis for the stereo- and enantioselectivity.

The crystal structure of the Fab-ferrocene complex was determined to $1.95 \AA$ resolution with good stereochemistry ( $\mathrm{Ta}$ ble 1). The overall structure is very similar to other known Fab structures (Fig. 2). The elbow angle (14) of $146.5^{\circ}$ is in the middle of the observed range $\left(127^{\circ}\right.$ to $\left.227^{\circ}\right)$ for antibodies (15). The inhibitor is completely buried (99\%) in the antibody combining site with $231 \AA^{2}$ of its $233 \AA^{2}$ surface area (14) being inaccessible to solvent, as has been found for the binding of other small haptens such as a chorismate mutase transition state analog (>90\%) and fluorescein $(94 \%)(10,16)$. In the antibody, $305 \AA^{2}$ of solvent-accessible surface is buried upon complexation $\left(44 \%\right.$ of $\mathrm{V}_{\mathrm{L}}$ and $56 \%$ of $\mathrm{V}_{\mathrm{H}}$ ) (Fig. 3).

Three hydrogen bonds are made from the cyclopentadienyl metal complex (inhibitor) to the antibody (Fig. 4). The Tyr ${ }^{\mathrm{L} 36}$ residue (position prefixes $\mathrm{L}$ and $\mathrm{H}$ designate light and heavy chains, respectively) forms a short hydrogen bond of $2.5 \AA$ with the carbonyl oxygen of the (dimethylamino)carbonyl group; this ferrocenyl amide mimics the N,N-dimethylacrylamide found within dienophile 2 . Two hydrogen bonds are formed from the ferrocenyl carboxylic acid (this functionality represents the carbamate found on diene 1 ) to the side chain amino group of $A s n^{\mathrm{L} 91}(2.9 \AA)$ and to the carboxyl of $\mathrm{Asp}^{\mathrm{H} 50}$. The hydrogen bond to $\operatorname{Asp}^{\mathrm{H} 50}(2.5 \AA)$ requires either the aspartic acid or the ferrocene carboxyl group to be protonated. At the $\mathrm{pH}$ of crystallization
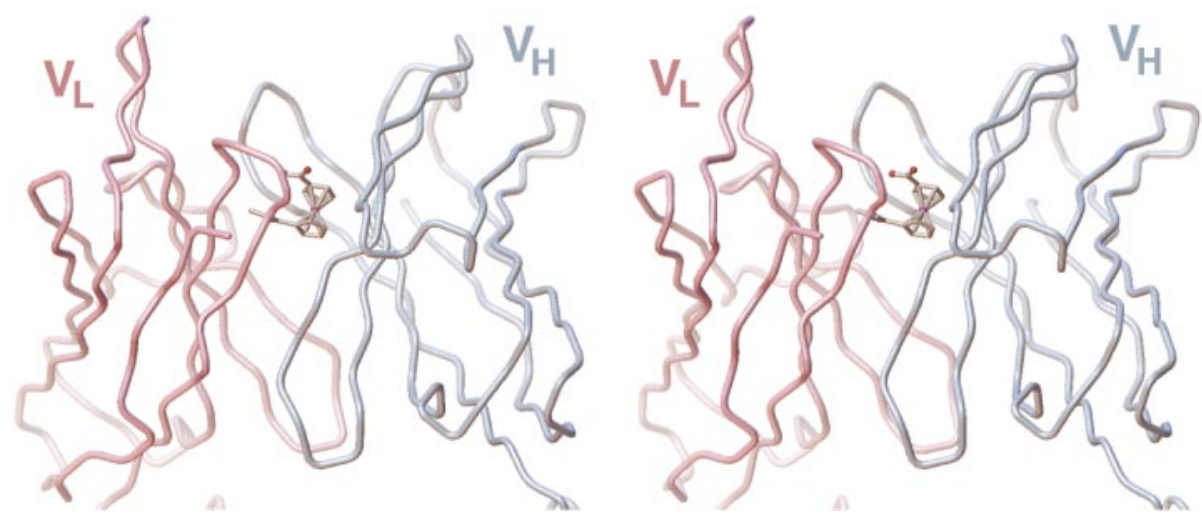

Fig. 2. Stereoview of the catalytic antibody Fab $13 \mathrm{G} 5$ in complex with the ferrocene compound $\mathbf{9}$ showing the inhibitor deeply bound in the antibody combining site. The side view shows the variable light chain $\left(V_{L}\right)$ on the left in magenta and the variable heavy chain $\left(V_{H}\right)$ on the right in cyan, with the hapten in ball-and-stick representation. Figures 2, 3, and 6 were produced with AVS (31).

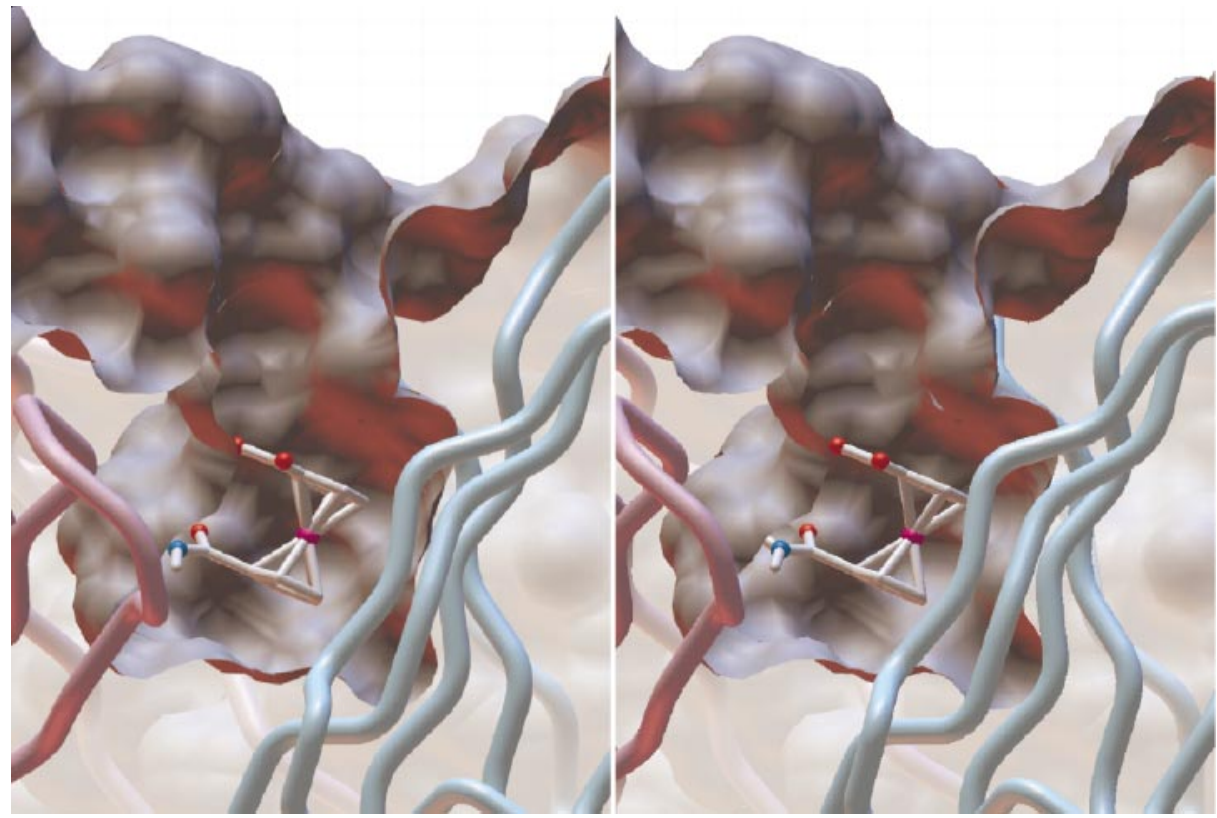

Fig. 3. Stereoview of the antibody combining site, slicing through the molecular surface of the 13G5 variable domain (1.5 $\AA$ sphere radius) and $\mathbf{9}$. The cyclopentadienyl ferrocene complex $\mathbf{9}$ is deeply buried in the antibody combining site and shielded from excess solvent. The $\mathrm{C} \alpha$ trace of the light chain is superimposed on the left in magenta and the heavy chain on the right in cyan. This close-up view shows the ferrocene inhibitor bound in a deep pocket with a narrow entrance through which the linker of the actual hapten $\mathbf{7}$ used for immunization could extend. 
form another hydrogen bond to the pyrrole nitrogen atom of the indole ring of framework residue $\mathrm{Trp}^{\mathrm{H} 47}$. Residue $\mathrm{Tyr}^{\mathrm{L} 36}$, which is directed toward the dienophile portion of the inhibitor, is oriented by an additional hydrogen bond from its side chain hydroxyl to the main-chain amide of Gly ${ }^{100 B}$. A water molecule $\mathrm{O}^{87}$ interacts directly with the inhibitor and forms a hydrogen bond (3.0 $\AA$ ) with the carboxyl side chain of the cyclopentadienyl ring. Additional bridging water hydrogen bonds are formed with $\mathrm{Asn}^{\mathrm{L} 91}$ and Gly ${ }^{\mathrm{H} 100 \mathrm{~A}}$ (3.2 $\AA$ and $2.9 \AA$, respectively).

A total of 45 van der Waals interactions (17 with $\mathrm{V}_{\mathrm{L}}, 28$ with $\mathrm{V}_{\mathrm{H}}$ ) are made between the antibody and the ferrocene inhibitor. These interactions mainly reside within the residues of the complementarity-determining region (CDR) loops [L1 (3), L3 (10), H1 (4), H2 (6), and H3 (13)] as expected (Fig. 2). No interactions are made with CDR L2 as is usually observed when small haptens are antigens (15).

The unbound cyclopentadienyl ferrocene 9 crystal structure (Fig. 5) contains two molecules of 1-carboxy-1'-[(dimethylamino)carbonyl]ferrocene 9 in the asymmetric unit (18). Bond lengths and angles are comparable to a similar metalloligand, ferrocene-1,1'-dicarboxylic acid, for which highly accurate low-temperature x-ray data and neutron studies are available (19). In each molecule of 9 , the $1,1^{\prime}$-substituents from the cyclopentadienyl rings are oriented in an anti conformation that minimizes steric intramolecular interactions. In one of these molecules, both of the disubstituents are nearly coplanar with the cyclopentadiene ring [torsion angles of 9.6 $6^{\circ}$ N1-C11$\mathrm{C} 1-\mathrm{C} 2)$ and $8.2^{\circ}$ (C5-C1-C11-O1)]. In the second molecule, the (dimethylamino)carbonyl group is rotated out of plane [torsion angles of $34^{\circ}\left(\mathrm{C} 5^{\prime}-\mathrm{C} 1^{\prime}-\mathrm{C} 11^{\prime}-\mathrm{O} 1^{\prime}\right)$ and $\left.45.4^{\circ}\left(\mathrm{C}^{\prime}-\mathrm{C} 1^{\prime}-\mathrm{C} 11^{\prime}-\mathrm{N} 1^{\prime}\right)\right]$, and the carbonyl oxygen forms a hydrogen bond to the carboxyl group of a symmetry-related molecule (Fig. 5). A second hydrogen bond is formed between the carbonyl oxygen of molecule 1 and the carboxyl group of molecule 2 (Fig. 5). In the ferrocene-1,1'dicarboxylic acid structure, the two acid groups are coplanar with the cyclopentadienyl rings and form hydrogen bonds with neighboring molecules. The energy barrier for rotation of the rings with respect to each other is very low in solution $(\sim 2$ to $5 \mathrm{kcal} / \mathrm{mol}$ ) (13). However, in the crystalline state, the formation of hydrogen bonds seems to determine the relative ar- rangement of the side groups.

In the antibody-antigen complex, the ferrocene ring rotation is restricted because of steric constraints and specific interactions with the antibody. The position and orientation of the substituents on 9 are determined by maximizing the number of hydrogen bonds that the ferrocene inhibitor can form with the protein (Fig. 4). Thus, the antibody is able to trap one conformer of the cyclopentadienyl compound. The electron density for $\mathbf{9}$ at this resolution is

Fig. 5. The crystal structure of 1 -carboxy-1'-[(dimethylamino)carbonyl]ferrocene $\mathbf{9}$. Molecules $\mathbf{1}$ and $\mathbf{2}$ are within the asymmetric unit, and $\mathbf{1 a}$ is a symmetry-related molecule. The molecules are connected by a hydrogen-bonding scheme between the carboxylate and the $N, N^{\prime}$-dimethylformamide that results in an anti conformation of the $1,1^{\prime}$-substituents.
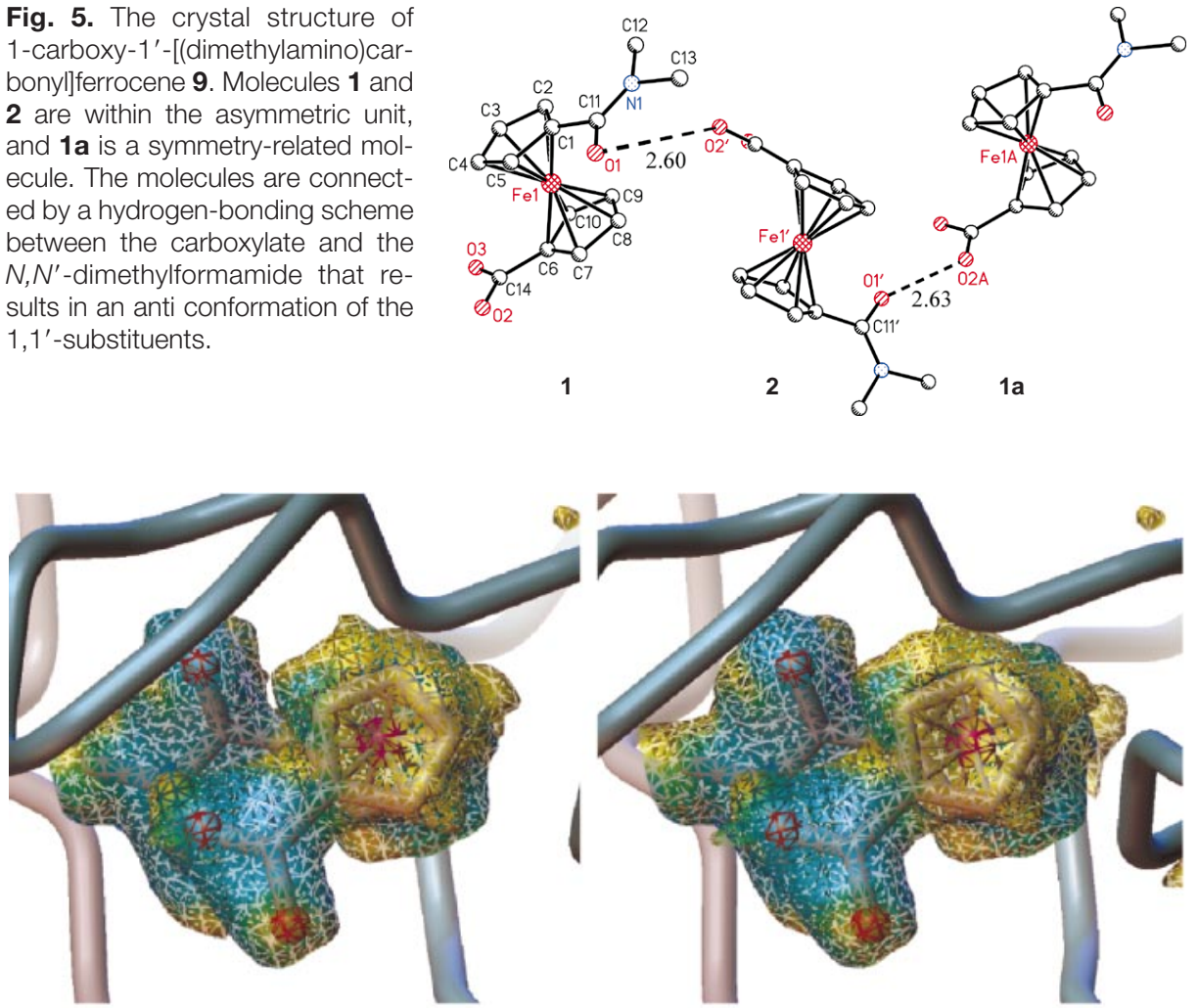

Fig. 6. Omit electron density map $\left(F_{\circ}-F_{c}\right)$, contoured at an interval of $1.1 \sigma$, of $\mathbf{9}$ in the Fab complex. The map has been colored according to the gradient of the density (32) in increasing order (yellow, green, cyan, purple), showing the highest gradient in purple surrounding the iron atom. The final ferrocenyl inhibitor model is superimposed on the density. The close-up view from the top into the antibody combining site shows the surrounding light chain in magenta and the heavy chain in cyan.

Fig. 4. Specific interactions of $\mathbf{9}$ with side chains of the Fab $13 G 5$ in the antibody-antigen complex. Three hydrogen bonds are formed with Fab residues Tyr ${ }^{\mathrm{L} 36}$, $\mathrm{Asp}^{\mathrm{H} 5 \mathrm{O}}$, and $\mathrm{Asn}{ }^{\mathrm{L} 91}$. In addition, a water molecule $\mathrm{O}^{87}$ is in close contact to ferrocene $\mathbf{9}$. The hydrogen-bonding network in the Fab combining site that orients the side chains of Tyr ${ }^{\mathrm{L} 6}$, $\mathrm{Asp}^{\mathrm{H} 50}$, and $\mathrm{Asn} \mathrm{n}^{\mathrm{L91}}$ in position to form hydrogen bonds with $\mathbf{9}$ are shown in (B). For Asn ${ }^{\mathrm{L} 91}$, this network is extended over three residues. Oxygen atoms

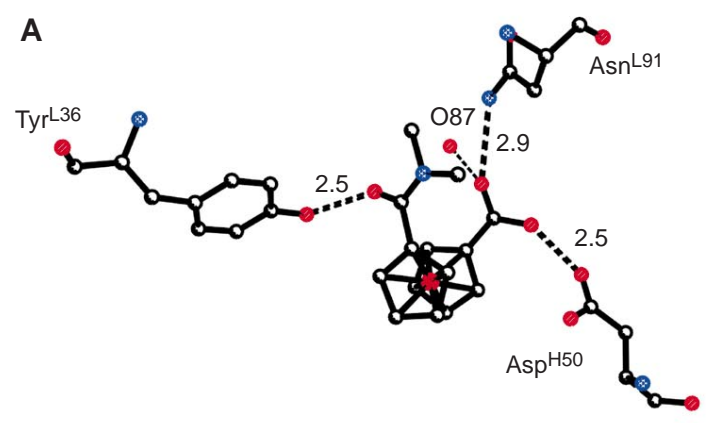

$B$

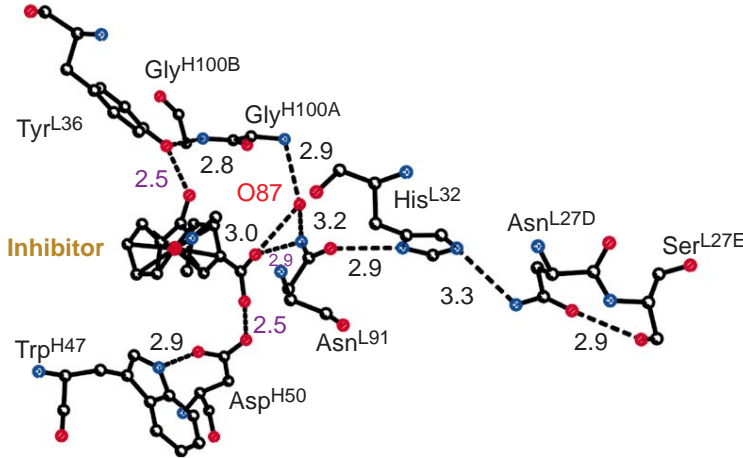

are shown in red and nitrogen atoms in blue. Figures 4 and 5 were produced with XP (31). 
excellent and gives no indication of disorder or multiple conformers for either of the 1,1'-substituents (Fig. 6). The eclipsed stacking appears energetically less favorable than the antiperiplanar conformation seen with 9 (Fig. 5), but it is compensated for by formation of hydrogen bonds to the Fab, similar to the ferrocene-1,1'-dicarboxylic acid structure (19), as well as by multiple hydrophobic interactions. The (dimethylamino)carbonyl group is slightly rotated out of the ring plane with torsion angles of $-27.7^{\circ}$ (C5-C1-C11-O1) and $-23.6^{\circ}$ (C2C1-C11-N1), which are less than in the second molecule of the small molecule structure. Again, these torsion angles ap-

Table 1. Crystallization, data collection, structure solution, and refinement for the antibody-hapten complex. Antibody 13G5 (immunoglobulin G1, к) was digested to a Fab with mercuripapain, which had been activated by incubation with cysteine and EDTA as described (26). Crystals were grown in 14\% MPEG 2000, $0.1 \mathrm{M}$ cacodylate at pH 6.5 in sitting drops by the vapor diffusion method. Crystalline plates of moderate size $\left(0.33\right.$ by 0.17 by $0.03 \mathrm{~mm}^{3}$ ) were obtained after a 2-day equilibration period and addition of $100 \mathrm{mM} \mathrm{NaCl}$ to the well solution. The light-sensitive ferrocenyl complex $\mathbf{9}$ was soaked into preformed crystals for 12 hours in the dark. A 9-Fab 13 G5 data set was collected at beamline 7-1 at the Stanford Synchrotron Radiation Laboratory (SSRL) on a Mar image plate detector at $-176^{\circ} \mathrm{C}$ with $30 \%$ glycerol as cryoprotectant. Data were processed with DENZO (27) and SCALEPACK (27). The Fab structure was determined by molecular replacement [AMORE (27)], with the native Fab (28) as the search model. A rigid-body refinement of the individual domains was performed in X-PLOR (27) from 10 to $4 \AA$, with subsequent multiple rounds of positional refinement in increasing shells of data from 10 to $3 \AA$ up to 10 to $1.95 \AA$ with $R=35.5 \%$ for this resolution range. Refinement was continued with the program SHELXL-96 (27). Multiple rounds of model building with the program $\mathrm{O}$ (27) followed by refinement were performed with standard restraints on bond distances and angles and a SIMU (assuming similar $B$ values for spatially adjacent atoms) restraint of 0.1 for $B$ values. For model building, $\sigma_{\mathrm{A}}$-weighted (27) $3 F_{\mathrm{o}}-2 F_{\mathrm{C}}$ and $F_{\mathrm{o}}-F_{\mathrm{C}}$ maps were calculated as implemented in SHELXPRO (27) as well as $2 F_{\circ}-F_{c}$ and $F_{\circ}-F_{c}$ shake omit maps, omitting $\sim 10 \%$ of the residues, with X-PLOR (27). In the final stages, hydrogen atoms were placed in calculated positions for Fab residues and refined without use of additional parameters. For the transition state analog, the iron atom was positioned into density, and after one additional round of refinement, density was clearly visible for the complete inhibitor. The inhibitor was built into difference electron density and refined with geometric restraints on bond lengths and angles. Distances were chosen from the small molecule structure and similar structures reported in the literature (19). The cyclopentadienyl rings and amide group were restrained to be planar. Rotation around the ring-center iron axis and out-of-plane rotation of the dimethylacrylamide group was not restricted. An overall anisotropic scaling and diffuse solvent correction was applied. The overall electron density was very clear, allowing precise positioning of side chains, except for an external loop region in the constant heavy chain $(\mathrm{H} 129$ to $\mathrm{H} 135)$, which is frequently disordered in other antibody structures (29). Analysis of the stereochemical quality of the model shows $92.9 \%$ of all residues are in the most favored regions and only one residue (Met ${ }^{L 51}$ ) is in a disallowed region, but it is in a $\gamma$ turn, as commonly observed in other antibody structures (30).

Crystallographic parameter

Value

Space group

Unit cell dimensions

Matthews constant $\left(\AA^{3} /\right.$ dalton $)$

Resolution range $(\AA)$

Observations

Unique reflections

Completeness (\%)

$1 / \sigma_{,}^{\dagger}$

$R_{\text {merge }}(\%) \neq$

Refined residues $(N)$

Refined water molecules $(N)$

Refinement range $(\AA)$

$R_{\text {cryst }}(\%) \S$

$R_{\text {free }}(\%) \|$

\section{Bond lengths $(\AA)$}

Bond angles $\left({ }^{\circ}\right)$

Dihedral angles $\left(^{\circ}\right)$

Improper angles $\left(^{\circ}\right)$

Average $B$ value $\left(\AA^{2}\right)$

Waters $\left(\AA^{2}\right)$

Ferrocene inhibitor $\left(\AA^{2}\right)$
Refinement statistics

C2

$a=182.9 \AA, b=40.5 \AA, c=67.7 \AA, \beta=109.0^{\circ}$

2.37

40.0-1.95

188,444

32,813

$95(84)^{*}$

$30.5(10.1)^{\star}$

$3.3(12.3)^{\star}$

435

309

10.0-1.95

$17.8\left(28,940 F_{0}>4 \sigma F_{0}\right)$

$18.4\left(30,944 F_{\circ} \geq 0\right)$

$25.3\left(1520 F_{0}>4 \sigma F_{0}\right)$

$26.5\left(1628 F_{0}^{\circ} \geq 0\right)$

Deviations from ideal geometry (rms)

0.006

1.72

29.9

1.71

$B$ values

22.0

28.1

15.3

*Values for data in outer shell of 2.02 to $19.5 \AA$. tThe signal-to-noise ratio. $\quad$ क $R_{\text {merge }}=\Sigma\left|F^{2}-\overline{F^{2}}\right| /$ $\Sigma F_{0}^{2} . \quad \$ R_{\text {cryst }}=100 \times \Sigma\left|F_{0}-F_{\mathrm{c}}\right| / \Sigma\left|F_{\mathrm{o}}\right|$, where $F_{\mathrm{o}}$ and $F_{\mathrm{c}}$ are the observed and calculated structure factors, respectively. ${ }^{\text {ret }} \| R_{\text {free }}$ is calculated in the same manner as $R_{\text {cryst }}{ }^{c}$, but from $5 \%$ of the data that was not used for refinement.

pear to be restricted by formation of a hydrogen bond.

The ferrocene inhibitor 9 acts as a loose mimic for a very early transition state of the Diels-Alder reaction, where the (dimethylamino)carbonyl group of the ferrocene represents the monosubstituted dienophile and the carboxyl group corresponds to the side chain of the diene (Fig. 1). Three hydrogen bonds constrain the orientation of the 1,1' disubstituted ferrocene 9 in the Fab complex. When the actual transition state for the substrates of the reaction is reached (which is closer to the geometry of the product), the main-chain amide group of Gly ${ }^{\mathrm{H} 100 \mathrm{~B}}$ and the water molecule $\mathrm{O}^{87}$ might also be available for hydrogen bonding to the dienophile. We suggest that, in the transition state of the reaction, the carbonyl oxygen of the amide forms a hydrogen bond to the $\mathrm{OH} \eta$ group of $\mathrm{Tyr}^{\mathrm{L} 36}$, and the $\mathrm{NH}$ of the diene carbamate forms a hydrogen bond to $\mathrm{Asp}^{\mathrm{H} 50}$, resulting in increased charge transfer and catalytic rate enhancement of the diene-dienophile.

Studies of solvent effects and theoretical calculations on the Diels-Alder reaction have shown that the $\mathrm{C}^{+}-\mathrm{O}^{-}$polarization of the carbonyl group of the dienophile is enhanced in the transition state. This enhanced polarization leads, for example, to a stronger hydrogen bond at the carbonyl oxygen of methyl vinyl ketone in the reaction with cyclopentadiene and results in a rate acceleration in water (3). The energy gain is about 1.5 to $2.0 \mathrm{kcal}$ per hydrogen bond, with an average number of hydrogen bonds per dienophile of 2 to 2.5 (3). The lowest energy transition state has a water hydrogen bonded to the methyl vinyl ketone carbonyl, syn to the double bond (3). These energy differences are small $(0.2$ to $0.8 \mathrm{kcal} / \mathrm{mol})$ (3), but the same preference is observed in the hydrogen bond formation of the transition state analog 9 with $\mathrm{Tyr}^{\mathrm{L} 36}$ in our structure and in the modeled exo transition states, where the hydrogen bond is on the same side as the double bond of the dienophile (Figs. 4 and 7).

The carboxylate substituent on ferrocene 9 is fixed by the interaction with $\mathrm{Asn}^{\mathrm{L} 91}, \mathrm{Asp}^{\mathrm{H} 50}$, and water $\mathrm{O}^{87}$ (Fig. 4). In addition to the hydrogen bonds, 45 van der Waals interactions are made between inhibitor and antibody 13G5. From the 17 formed with $\mathrm{V}_{\mathrm{L}}$, only two involve carbon atoms (C1 and C5) of the cyclopentadienyl rings, whereas the remaining interactions are almost exclusively with the (dimethylamino)carbonyl group on 9. These interactions severely restrict rotation about the ring-iron-ring axis, because the (dimethylamino)carbonyl appendage would collide with neighboring protein atoms. However, the carboxylic acid on 9 appears to be steri- 
cally less restricted in the binding site so that there is enough space in the binding site for rotation of the upper ring by about one ring-carbon position (one-fifth rotation or $\sim 72^{\circ}$ ) without interfering with Fab atoms. Hence, it appears that hydrogen bonding from the Fab to the inhibitor carboxylate fixes the relative orientation of the upper ring. Further rotation is restricted because of steric hindrance with the mainchain atoms of residues $\mathrm{Gly}^{\mathrm{H} 35}, \mathrm{Ala}^{\mathrm{H} 93}$, Gly ${ }^{\mathrm{H} 100 \mathrm{~B}}$, and the side chain of $\mathrm{Asp}^{\mathrm{H} 50}$. Also, the staggered conformation, as seen in the small molecule 9, is not possible because of the position of $\mathrm{Ala}^{\mathrm{H} 93}$. The heavy chain forms most of its van der Waals contacts with the cyclopentadienyl rings; the cyclopentadienyl ring mimicking the diene functionality makes 17 contacts and the corresponding dienophile ring makes 11 contacts. Thus, extensive hydrophobic contacts with the Fab play an important role in hapten binding and form a suitable environment for a bound hydrophobic ligand.

In the actual hapten 7 used to elicit the antibody, the carboxylate of $\mathbf{9}$ is replaced by a (4-carboxy-1-oxylbutyl)amino appendage (Fig. 1). This functionality can be compared to the N-butadienyl-carbamic acid that was used as a model compound for the DielsAlder reaction described previously (12). The question arises as to how the antibody accommodates the actual hapten used for immunization, and which, if any, of the carboxyl group oxygens of 9 correspond to either the nitrogen or the carbonyl oxygen of the carbamate 7 . This hapten can be mod- eled onto the ferrocene derivative by overlapping the lower "dienophile" ring and maintaining the position of the (dimethylamino)carbonyl group. The upper "diene" ring now becomes more restricted in the binding site because of the bulkier (4-carboxy-1-oxylbutyl)amino functionality but still can be modeled in both exo and endo conformations. However, only the exo conformer can form hydrogen bonds with the Fab. In this conformation, $\mathrm{Asp}^{\mathrm{H} 50}$ can form a hydrogen bond $(\sim 3 \AA)$ with the amine, and $A s n^{L 91}$ hydrogen bonds to the carbonyl oxygen $(\sim 2.5 \AA)$. In the structure of 9 , the carboxyl oxygen that hydrogen bonds to $\mathrm{Asp}^{\mathrm{H} 50}$ corresponds to the amide nitrogen of 7 , and the second carboxyl oxygen, in contact with $A s n^{\mathrm{L} 91}$, is equivalent with the carbonyl oxygen of the carbamate. Thus, equivalent hydrogen bonds can be formed with the same Fab residues to the carbamate on 7 as with the carboxylate of $\mathbf{9}$.

$\mathrm{Ab}$ initio calculations suggest that two of these three hydrogen-bonding interactions described above result in transition state stabilization. The extent of catalysis possible from these interactions was determined from quantum mechanical investigations of the transition states of the Diels-Alder reaction of $N, N$-dimethylacrylamide with $N$-butadienyl carbamic acid, a model for diene 1 . The geometries of the reactants and transition states were optimized with RHF/3-21G ab initio calculations $(20,21)$, and energies were evaluated with density functional theory at the Becke3LYP/6-31G* level $(22,23)$.

One exo transition state of the reaction
(Fig. 7), and those obtained when the acrylamide is hydrogen-bonded to a water molecule or when the carbamate $\mathrm{NH}$ is hydrogenbonded to a formate constitute model systems in which water corresponds to the side chain $\mathrm{OH} \eta$ of $\mathrm{Tyr}^{\mathrm{L} 36}$ and formate mimics the carboxyl group of $\mathrm{Asp}^{\mathrm{H} 50}$. The hydrogen bond between water and the acrylamide stabilizes the complex by $6.3 \mathrm{kcal} / \mathrm{mol}$; this interaction lowers the lowest unoccupied molecular orbital and increases the reactivity of the dienophile. In the transition state, the strength of the hydrogen bond increases to $7.4 \mathrm{kcal} / \mathrm{mol}$, resulting in a decrease of 1.1 $\mathrm{kcal} / \mathrm{mol}$ in the activation energy of the reaction. However, the hydrogen bond between acrylamide and the side chain $\mathrm{OH} \eta$ of $\mathrm{Tyr}^{\mathrm{L} 36}$ by itself is unlikely to account for most of the catalytic effect observed for 13G5 because the uncatalyzed reaction occurs in aqueous solution where hydrogen bonds between acrylamide and water also result in transition state stabilization.

The formation of a short, strong hydrogen bond between formate and the $\mathrm{NH}$ of the diene carbamate substituent actually results in a much larger activation energy decrease of $5.9 \mathrm{kcal} / \mathrm{mol}$ (Fig. 7C). Thus, a hydrogen bond between the $\mathrm{NH}$ of the diene carbamate and the carboxylate of $\mathrm{Asp}^{\mathrm{H} 50}$ in $13 \mathrm{G} 5$ is predicted to be more important than the hydrogen bond to the dienophile in inducing rate acceleration.

These hydrogen-bonding interactions each cause increased asynchronicity and charge separation in the transition state. When both are present (Fig. 7D), the ef-

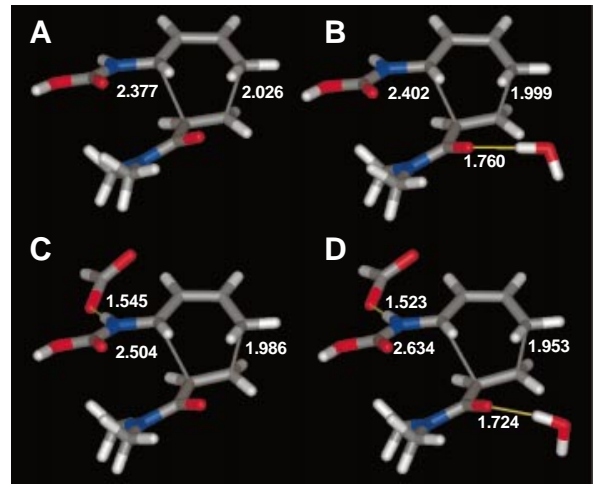

Fig. 7. $\mathrm{RHF} / 3-21 \mathrm{G}$ transition structures for the exo Diels-Alder reaction of $N, N$-dimethylacrylamide and $N$-butadienyl carbamic acid without $(\mathbf{A})$ and with (B) water hydrogen-bonded to the dienophile carbonyl oxygen, with formate hydrogen-bonded to the diene $(\mathbf{C})$, and with hydrogen bonds to both water and formate (D). Activation energies for (A), (B), (C), and (D) are 22.2, 21.1, 16.3, and 12.2 $\mathrm{kcal} / \mathrm{mol}$, respectively, and are from Becke3LYP/6$31 \mathrm{G}^{*} / / \mathrm{HF} / 3-21 \mathrm{G}+\mathrm{HF} / 3-21 \mathrm{G}$ ZPE calculations (20-23). All bond lengths are in angstroms, and oxygen atoms are in red, nitrogen atoms in blue, and carbon atoms in white. Figures 7 and 8 were produced with MOLMOL (31).

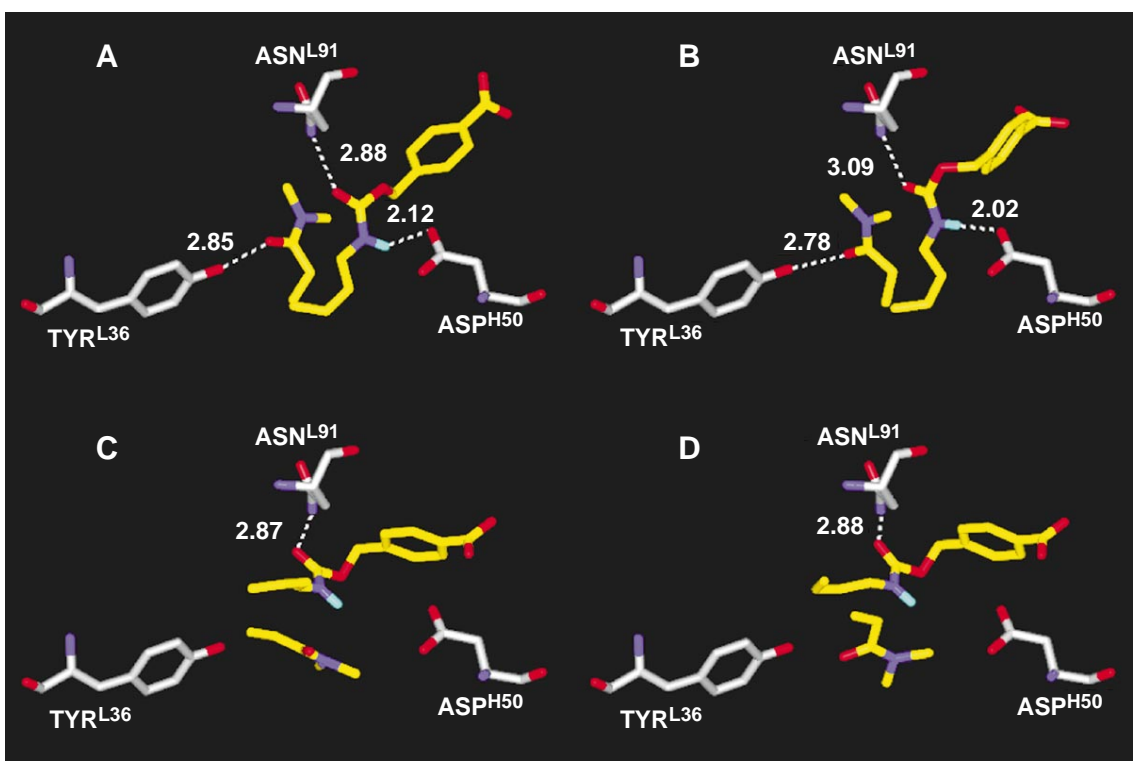

Fig. 8. Favored orientations for each of the four isomeric transition states in antibody $13 \mathrm{G} 5$ predicted by AUTODOCK: (A) $(3 R, 4 R)$-exo, (B) $(3 S, 4 S)$-exo, (C) $(3 R, 4 S)$-endo, and (D) $(3 S, 4 R)$-endo. The lowest energy structures in $(A),(C)$, and (D), and the second lowest in (B) are shown. Only the transition states of the reaction (Fig. 1) and the interacting side chains that form hydrogen bonds in the crystal structure of the ferrocenyl inhibitor are shown for clarity. Only in the exo forms are all three hydrogen bonds made that are observed in the crystal structure of the inhibitor-Fab complex. 
fects are cooperative and the activation energy is decreased by $10 \mathrm{kcal} / \mathrm{mol}$ relative to the uncatalyzed reaction. Thus, the presence of both hydrogen bonds is required for maximum rate acceleration. If only a subset of the stereoisomeric Diels-Alder transition states is capable of binding in orientations that preserve both of these hydrogen bonds, then both rate acceleration and stereospecificity are possible.

The Fab-ferrocene inhibitor complex structure provides a key to understanding the ability of $13 \mathrm{G} 5$ to achieve complete exo versus endo stereoselectivity and high enantioselectivity in a Diels-Alder reaction. We have explored the mechanism of this high specificity by investigating how each of the four possible transition states might interact with the Fab 13G5 combining site. The transition states were constructed from ab initio RHF/3-21G calculations on the reactions of $N$-butadienylcarbamate with $N, N$-dimethylacrylamide. One of the exo transition states is shown in Fig. 7. For the modeling studies, the acidic hydrogen of each transition state was replaced by a standard benzyl carboxylate side chain.

The four stereoisomeric transition states were docked (24) into the binding site of 13G5. The $(3 R, 4 R)$-exo transition state docks in a fashion roughly similar to 9 , with hydrogen bonds between $\mathrm{Tyr}^{\mathrm{L} 36}$ and the dienophile carbonyl group, Asp ${ }^{\mathrm{H} 50}$ and the $\mathrm{NH}$ of the diene carbamate, and $\mathrm{Asn}^{\mathrm{L} 91}$ with the carbonyl group of the carbamate. This docking mode provides both of the hydrogen bonds postulated to accelerate the Diels-Alder reaction. Although one might have expected that the transition state that most closely overlays the ferrocenyl complex 9 would be the most highly stabilized, docking calculations suggest that the $(3 R, 4 R)$-exo enantiomer should be stabilized most by hydrogen interactions with the antibody. The transition state that is closest to the ferrocenyl complex 9, $(3 S, 4 S)$-exo, can also dock in an orientation that preserves the two catalytic hydrogen bonds (Fig. 8B). However, the lowest energy docking mode for $(3 S, 4 S)$-exo lacks the activating hydrogen bonds to the diene amide and dienophile carbonyl. Both endo transition structures dock readily into the binding site (Fig. 8, C and D) but lack hydrogen bonds to the dienophile carbonyl oxygen or to the diene amide.

We conclude then that the exo transition states can be bound in orientations that allow formation of hydrogen bonds to both the diene and dienophile moieties. The strength of these hydrogen bond interactions is increased in the transition state because of charge transfer from diene to dienophile. The endo transition states bind in orientations that do not allow formation of these strong hydrogen bonds. The $(3 R, 4 R)$-exo transition structure appears to be the most highly stabilized, but definitive evidence can only be obtained by determination of the absolute configuration of the product obtained from the 13G5 catalysis (25).

The binding pocket of antibody 13G5 thus provides enough space to accommodate all four possible ortho transition states of the Diels-Alder reaction. However, only in the exo transition state can the same hydrogen bonds be formed that are seen in the structure of the ferrocene complex 9 . The role of $\mathrm{Tyr}^{\mathrm{L} 36}$ can be entirely attributed to rate enhancement, which is due to its Lewis acid effect on the dienophile, thus activating it for nucleophilic attack. Hydrogen bonding to $\mathrm{Asp}^{\mathrm{H} 50}$ decreases the activation energy even more because the negatively charged Asp makes the carbamate of the diene a much better electron donor and, together with $A s n^{\mathrm{L} 91}$, provides the structural framework for the antibody to orient and position the substrates for catalysis. A greater catalytic rate enhancement than the observed $k_{\text {cat }}$ of $1.20 \times 10^{-3} \mathrm{~min}^{-1}$ could be achieved by introduction of either a stronger or an additional electrophile within hydrogen-bonding distance to the dienophile. The resulting increased electron-withdrawing effect should enable a more efficient nucleophilic attack on the dienophile. Furthermore, mutation of $\mathrm{Asn}^{\mathrm{L} 91}$ to Asp and $\mathrm{Asp}{ }^{\mathrm{H} 50}$ to Asn might change the reaction pathway, shifting the catalysis toward the endo Diels-Alder reaction. Further kinetic and crystallographic analyses of these mutants with and without bound substrates should provide additional insights into this proposed reaction mechanism for a Diels-Alder reaction and in the evolution of protein catalysts for disfavored processes.

\section{REFERENCES AND NOTES}

1. A. Streitwieser and C. H. Heathcock, Introduction to Organic Chemistry (Macmillan, New York, 1981).

2. J. Sauer and R. Sustmann, Angew. Chem. Int. Ed. Engl. 19, 779 (1980); Y. Li and K. N. Houk, J. Am. Chem. Soc. 115, 7478 (1993)

3. D. C. Rideout and R. Breslow, J. Am. Chem. Soc 102, 7816 (1980); P. A. Grieco, P. Garner, Z. He Tetrahedron Lett. 24, 1897 (1983); J. F. Blake and W. L. Jorgensen, J. Am. Chem. Soc. 113, 7430 (1991); W. L. Jorgensen, J. F. Blake, D. Lim, D. L. Severance, J. Chem. Soc. Faraday Trans. 90, 1727 (1994); J. F. Blake, D. Lim, W. L. Jorgensen, J. Org. Chem. 59, 803 (1994).

4. P. G. Schultz and R. A. Lerner, Science 269, 1835 (1995); B. Lavey and K. D. Janda, in Antibody Engineering, H. Y. Wang and T. Imanaka, Eds. (American Chemical Society, Washington, DC,1995), pp. 123138; K. D. Janda, C. G. Shevlin, C.-H. L. Lo, in Comprehensive Supramolecular Chemistry, J. M. Lehn et al., Eds. (Pergamon, New York, 1996), pp. 43-73; E. Keinan and R. A. Lerner, Isr. J. Chem. 36, 133 (1996).

5. W. P. Jencks, Catalysis in Chemistry and Enzymology (McGraw-Hill, New York, 1969); Adv. Enzymol. 43, 219 (1975).

6. A. Tramontano, K. D. Janda, R. A. Lerner, Science 234, 1566 (1986); K. D. Janda, S. J. Benkovic, R. A Lerner, ibid. 244, 437 (1989); G. W. Zhou, J. Guo, W.
Huang, R. J. Fletterick, T. S. Scanlan, ibid. 265, 1059 (1994); J. Guo, W. Huang, G. W. Zhou, R. J. Fletterick, T. S. Scanlan, Proc. Natl. Acad. Sci. U.S.A. 92 1694 (1995); P. A. Patten et al., Science 271, 1086 (1996); B. Golinelli-Pimpaneau et al.. Structure 2 , 175 (1994); J.-B. Charbonnier et al., Science 275 1140 (1997)

7. P. Wirsching, J. A. Ashley, C-H. L. Lo, K. D. Janda, R. A. Lerner, Science 270, 1775 (1995); J. Wagner, R. A. Lerner, C. F. Barbas III, ibid., p. 1797; R. Björnestedt, G. Zhong, R. A. Lerner, C. F. Barbas, J. Am. Chem. Soc. 118, 11720 (1996).

8. K. D. Janda, C. G. Shevlin, R. A. Lerner, Science 259, 490 (1993); B. F. Cravatt, J. A. Ashley, K. D. Janda, D. L. Boger, R. A. Lerner, J. Am. Chem. Soc. 116, 6013 (1994).

9. H. Oikawa, K. Katayama, Y. Suzuki, A. Ichihara, J. Chem. Soc. Chem. Commun. 1995, 1321 (1995).

10. M. R. Haynes, E. A. Stura, D. Hilvert, I. A. Wilson, Science 263, 646 (1994); M. R. Haynes, A. Heine, I. A. Wilson, Isr. J. Chem. 36, 133 (1996).

11. D. Hilvert, K. W. Hill, K. D. Nared, M.-T. M. Auditor, J. Am. Chem. Soc. 111, 9261 (1989); A. C. Braisted and P. G. Schultz, ibid. 112, 7430 (1990).

12. V. E. Gouverneur et al., Science 262, 204 (1993)

13. J. T. Yli-Kauhaluoma et al., J. Am. Chem. Soc. 117 7041 (1995).

14. The elbow angle of a Fab is between the pseudotwofold rotation axes relating the variable $V_{L}$ and $V_{b}$ domains to the constant $\mathrm{C}_{\mathrm{L}}$ and $\mathrm{C}_{\mathrm{H}} 1$ domains and was calculated with the programs OVRLAP [M. G. Rossman and P. Argos, J. Biol. Chem. 250, 7525 (1975)] and ELBOW (D. H. Fremont and T. O. Yeates, personal communication). All molecular surface areas buried by interaction were calculated with the program MS [M. L. Connolly, J. Appl. Crystallogr. 16, 439 (1983)] with a $1.7 \AA$ A probe sphere and standard atomic radii [S. Sheriff, Immunomethods 3, 191 (1993)].

15. I. A. Wilson and R. L. Stanfield, Curr. Opin. Struct. Biol. 3, 113 (1993); ibid. 4, 857 (1994).

16. J. N. Herron, X. M. He, M. L. Mason, E. W. Voss, A. B. Edmundson, Proteins Struct. Funct. Genet. 5 271 (1989).

17. E. A. Kabat, T. T. Wu, H. M. Perry, K. Gottesman, C. Foeller, Sequences of Proteins of Immunological Interest (Department of Health and Human Services, National Institutes of Health, Bethesda, MD, ed. 5 , 1991)

18. The ferrocene derivative 9 was crystallized from acetone at $4^{\circ} \mathrm{C}$ in space group $P^{-1}$ with cell dimensions $a=5.99 \AA, b=12.41 \AA, c=17.29 \AA, \alpha=90.81^{\circ}$, $\beta=93.6^{\circ}, \gamma=93.34^{\circ}$. The structure was solved with Direct Methods and SHELXS (27) and refined with SHELXL (27).

19. F. Takusagawa and T. F. Koetzle, Acta Crystallogr. Sect. B 35, 2888 (1979); G. J. Palenik, Inorg. Chem. 8, 2744 (1969).

20. All ab initio and density functional theory (DFT) calculations were carried out with the Gaussian 94 program [M. J. Frisch et al., Gaussian 94 Revision D.2 (Gaussian, Inc., Pittsburgh, PA, 1995).

21. W. J. Hehre, L. Radom, P. v. R. Schleyer, J. A. Pople, Ab Initio Molecular Orbital Theory (Wiley-Interscience, New York, 1986), pp. 76-79.

22. A. D. Becke, J. Chem. Phys. 98, 5648 (1993); C Lee, W. Yang, R. G. Parr, Phys. Rev. B 37, 785 (1988).

23. Hybrid Hartree-Fock (HF)-DFT methods give excellent results for Diels-Alder reactions. See, for example, V. Barone and R. Arnaud, J. Chem. Phys. 106 8727 (1997); B. R. Beno, K. N. Houk, D. A. Singleton, J. Am. Chem. Soc. 118, 9984 (1996); E. Gold stein, B. Beno, K. N. Houk, ibid., p. 6036, and references therein.

24. Each of the four transition states was docked with Fab 13G5 by using the program AUTODOCK [D. S Goodsell and A. J. Olson, Proteins Struct. Funct. Genet. 8, 195 (1990); D. S. Goodsell, G. M. Morris, A. J. Olson, J. Mol. Recognit. 9, 1 (1996)]. This program uses simulated annealing to locate low-energy binding modes; the antibody structure is held fixed in the $x$-ray geometry. Similar results from the docking experiments were obtained with coordinates of both the unliganded antibody (28) and the inhibitor-bound antibody. Charges on the transition state atoms 
were obtained with AM1 semi-empirical calculations. The positions of the transition states were varied starting from 100 different, randomly chosen starting orientations in a $22.5 \AA$ cube encompassing the antibody variable region. Each simulation involved 150 cycles of simulated annealing. Five flexible dihedral angles of the diene substituent in each transition state were allowed to vary. The energy of interaction was calculated with a potential function composed of van der Waals and Coulombic terms. As a check on this procedure, the ferrocene inhibitor 9 was docked into the antibody; AUTODOCK reproduces the binding position found experimentally to a root mean square (rms) of $0.6 \AA$.

25. C. Cannizzaro and K. N. Houk, personal communication

26. E. A. Stura, G. G. Fieser, I. A. Wilson, Immunomethods 3, 164 (1993)

27. The $x$-ray crystallographic programs and methods used were the following. (i) DENZO and SCALEPACK: Z. Otwinowski, in Data Collection and Processing, L. Sawyer, N. Isaacs, S. Bailey, Eds. (SERC Daresbury Laboratory, Warrington, UK, 1993), p. 56; (ii) MERLOT: P. M. D. Fitzgerald, J. Appl. Crystallogr. 21, 273 (1988); (iii) AMORE: J. Navaza, Acta Crystallogr. A50, 157 (1994); (iv) O: T. A. Jones, J.-Y. Zou, S. W. Cowan, M. Kjeldgaard, ibid. A47, 110 (1991); (v) X-PLOR: A. T. Brünger, J. Kuriyan, M. Karplus, Science 235, 458 (1987); A. T. Brünger, X-PLOR, Version 3.1: A System for $X$-ray and NMR ( Yale Univ. Press, New Haven, CT, 1992); (vi) SHELXL,SHELXS,SHELXPRO: G. M. Sheldrick, SHELXL-96, Program for crystal structure refinement, University of Göttingen (1996); (vii) sigma-A: R. J. Read, Acta Crystallogr. A42, 140 (1986)
28. A. Heine, E. A. Stura, K. D. Janda, I. A. Wilson, unpublished data.

29. R. L. Stanfield, T. M. Fieser, R. A. Lerner, I. A. Wilson, Science 248, 712 (1990)

30. J. H. Arevalo, E. A. Stura, M. J. Taussig, I. A. Wilson, J. Mol. Biol. 231, 103 (1993).

31. The graphics programs used were the following. (i) AVS: C. Upson et al., IEEE Comput. Graph. Appl. 9 30 (1989); (ii) XP: SHELXTL 5.03, Siemens Industrial Automation, Inc., Madison, WI, 1990-1995 MOLMOL: R. Koradj, M. Billeter, K. Wüthrich, J. Mol. Graph. 14, 51 (1996)

32. G. D. Purvis and C. Culberson, J. Mol. Graph. 4 (1986).

33. We thank M. Pique for production of figures, the Stanford Synchrotron Radiation Laboratory for data collection time, and the staff of beamline 7-1 for their assistance; G. Fieser and R. Stefanko for technical assistance, C.-H. Lo for Fab sequence determination, R. Stanfield and K. Gruber for discussions and comments, K. Renner for computational assistance, D. McRee for map generation in XFIT, R. Lerner for support and encouragement, and R. Chadha for data collection of 9 . Supported by $\mathrm{NIH}$ grants $\mathrm{PO} 1$ CA27489 (I.A.W. and K.D.J.) and GM-43858 (K.D.J.), NSF grant CHE9120588 (K.N.H.), and a fellowship by TEKES, Technology Development Centre, The Finnish Centre for International Mobility and Exchange Programmes, Helsinki, Finland (J.Y-K). This is publication 11111-MB from The Scripps Research Institute. The coordinates have been deposited in the Brookhaven databank with access code $1 \mathrm{a} 3 \mathrm{l}$ and are available immediately from wilson@scripps.edu or aheine@scripps.edu.

18 September 1997; accepted 30 January 1998

\section{RNA Folding at Millisecond Intervals by Synchrotron Hydroxyl Radical Footprinting}

\section{Bianca Sclavi, Michael Sullivan, Mark R. Chance, ${ }^{\star}$ Michael Brenowitz, ${ }^{\star}$ Sarah A. Woodson*}

Radiolysis of water with a synchrotron $\mathrm{x}$-ray beam permits the hydroxyl radical-accessible surface of an RNA to be mapped with nucleotide resolution in 10 milliseconds. Application of this method to folding of the Tetrahymena ribozyme revealed that the most stable domain of the tertiary structure, P4-P6, formed cooperatively within 3 seconds. Exterior helices became protected from hydroxyl radicals in 10 seconds, whereas the catalytic center required minutes to be completely folded. The results show that rapid collapse to a partially disordered state is followed by a slow search for the active structure.

The speed of chemical reactions carried out by ribozymes is often limited by conformational changes in the RNA (1). As a result, the process by which RNA molecules fold into their native conformation has received much attention. Early investigations

B. Sclavi, M. Sullivan, M. R. Chance, Department of Physiology and Biophysics, Center for Synchrotron Biosciences, Albert Einstein College of Medicine of Yeshiva University, 1300 Morris Park Avenue, Bronx, NY 10461, USA.

M. Brenowitz, Department of Biochemistry, Center for Synchrotron Biosciences, Albert Einstein College of Medicine of Yeshiva University, 1300 Morris Park Avenue, Bronx, NY 10461, USA.

S. A. Woodson, Department of Chemistry and Biochemistry, University of Maryland, College Park, MD 207422021, USA.

*To whom correspondence should be addressed. into the folding of tRNA established approximate time scales for the formation of RNA secondary $\left(10^{-4}\right.$ to $\left.10^{-5} \mathrm{~s}\right)$ and tertiary interactions $\left(10^{-2}\right.$ to $\left.10^{-1} \mathrm{~s}\right)$, with the reorganization of incorrect secondary structures occurring more slowly ( 0.1 to 1 s) (2). Recent work has shown that folding of large RNAs is more complex (3), involving multiple pathways (4). Individual domains of an RNA may form at rates that differ by orders of magnitude, with some transitions requiring minutes to reach completion (3-7). Identification of the paths by which large RNAs fold has been hampered by the lack of experimental methods capable of probing RNA conformation with nucleotide resolution at subsecond time scales (8). Here, we describe direct measurement of the complete folding pathway of the Tetrahymena ribozyme by hydroxyl radical footprinting using a synchrotron $\mathrm{x}$-ray beam.

Hydroxyl radical ribose oxidation and resulting strand cleavage are correlated with the solvent accessibility of the RNA backbone $(9,10)$ and are insensitive to base sequence and secondary structure (11). Generation of hydroxyl radicals by the radiolysis of water yields cleavage products that are comparable with Fe(II)-EDTA-dependent reactions $(7,12)$. The high flux provided by white-light $\mathrm{x}$-ray beams at the National Synchrotron Light Source (NSLS) permits footprinting of the ribozyme to be accomplished with millisecond time resolution (7).

The ribozyme derived from the Tetrahymena group I intron (Fig. 1A) folds into a well-defined tertiary structure in the presence of $\mathrm{Mg}^{2+}$, and $\mathrm{Mg}^{2+}$ is required for catalytic activity (1). The ribozyme contains at least three domains of tertiary structure (13) that, when separated, can reassociate to form the active ribozyme (14). The domain containing paired regions P4-P6 (Fig. 1A) folds independently $(10,15)$, and formation of P4-P6 has been proposed to be the first step in the folding pathway of the ribozyme $(3,16)$. In earlier experiments in which RNA was manually mixed with $\mathrm{Mg}^{2+}$ before exposure to the $\mathrm{x}$-ray beam, we showed that the tertiary structure of the P4-P6 domain is formed within $30 \mathrm{~s}$, the initial time of the assay (7).

To resolve early steps in the ribozyme folding pathway, we installed a stoppedflow apparatus with an $\mathrm{x}$-ray exposure chamber on NSLS beamline X-9A (17). The flux of X-9A absorbed by the sample was sufficient to cleave $20 \%$ of the RNA molecules with exposures as short as $10 \mathrm{~ms}$ (18). Folding reactions were begun by mixing RNA with buffer containing $\mathrm{Mg}^{2+}$, to a final concentration of $10 \mathrm{mM}$ (19). Samples were irradiated at a series of times after mixing, and the hydroxyl radical cleavage products were separated by gel electrophoresis (19). The ribozyme was fully active after passage through the stopped-flow apparatus (20), verifying that the RNA had folded correctly under these experimental conditions.

We determined the folding kinetics of the ribozyme by quantitating the changes in solvent accessibility of individual sites as a function of time (Fig. 2) (7,21). After the addition of $\mathrm{Mg}^{2+}$, specific nucleotides within the P4-P6 domain became protected from cleavage within $100 \mathrm{~ms}$ (22), and the extent of protection reached a plateau within several seconds (Fig. 2). Comparison of this plateau with control reactions, 